\title{
Time and Again, Children Resemble Their Parents
}

A often reminded of the genetic and behavioral similarities of offspring to their parents. Despite the children's unique characteristics, moulded by their genomic makeup and differing experience of their environment, even strangers can see family resemblances. The paper by Uesaka et al. (1) in the current edition of The Journal of Nuclear Medicine applies the same principle to PET to characterize the nature of focal ${ }^{18} \mathrm{~F}$-FDG abnormalities remote from primary non-small cell lung cancer. Although underpinned by some fairly complex postprocessing of data, the authors' basic message is that if the temporal profile of accumulation or washout of ${ }^{18} \mathrm{~F}$-FDG from a remote

\section{See page 1606}

site does not closely track that in the known primary, then it probably does not belong to the same process. Continuing the analogy, if the child does not look like the parents, it is time for paternity testing!

Using the temporal profile of tracer localization in a lesion as a means of characterizing its nature is almost as old as nuclear medicine imaging itself. In the 1950s, benign and malignant thyroid nodules were recognized to differ in their ability to take up and then excrete radioactive iodine. The concept of hot and cold thyroid nodules reflects the fact that the process of uptake of radioactive iodine is generally sufficient to dichot-

Received May 1, 2008; revision accepted May 5, 2008.

For correspondence or reprints contact: Rodney J Hicks, Centre for Molecular Imaging, Peter MacCallum Cancer Centre, 12 St. Andrew's Place, East Melbourne, Victoria 3002, Australia. E-mail: Rod.Hicks@petermac.org

COPYRIGHT @ 2008 by the Society of Nuclear Medicine, Inc.

DOI: 10.2967/jnumed.108.052142 omize benign lesions from those that could be malignant. However, with ${ }^{131} \mathrm{I}$ scanning some malignant lesions were identified as being "warm" because of on ongoing ability to organify radioactive iodine but impaired excretion relative to normal thyroid tissue, leading to relatively higher uptake in the nodule than in normal thyroid tissue at late time points. Although giving useful information about the biochemical function of different clones on thyroid cells, delayed imaging protocols were largely dropped in favor of the more pragmatic and patient-friendly early imaging approach. Other examples in nuclear oncology of dual-time-point imaging have been the use of ${ }^{201} \mathrm{Tl}$ to characterize brain, lung, and soft-tissue lesions (2,3). Many malignant tumors have high uptake and retention of ${ }^{201} \mathrm{Tl}$, whereas ${ }^{201} \mathrm{Tl}$ tends to wash out from inflammatory processes. However, again for pragmatic reasons many facilities currently perform either only early or delayed imaging.

Dual-phase ${ }^{18} \mathrm{~F}-\mathrm{FDG}$ PET has recently been promoted as a means to at least partially overcome the imperfect specificity of this technique, particularly with respect to lung nodules (4). The lack of specificity for the diagnosis of such nodules is imposed by the fact that ${ }^{18} \mathrm{~F}-\mathrm{FDG}$ is a tracer of glucose metabolism rather than of a process unique to cancer cells. Accordingly, various granulomatous and inflammatory processes can mimic cancer (5). Earlier experimental studies indicated that even within tumor lesions it is likely that inflammatory cells contribute in a minor degree to the ${ }^{18} \mathrm{~F}-\mathrm{FDG}$ signal $(6,7)$. However, it was recognized that, unlike cancer cells, inflammatory processes have progressive washout of ${ }^{18}$ F-FDG over time $(8,9)$. This is probably because many inflammatory cells have higher levels of glucose-6-dephosphorylase than do most cancers, which generally lack this enzyme. Thus, whereas cancers usually continue to accumulate ${ }^{18} \mathrm{~F}$-FDG up to 180 min after administration, the tracer tends to wash out from inflammatory lesions after reaching a peak at around 45-60 min. Accordingly, although scanning at between 45 and $60 \mathrm{~min}$, as generally performed for clinical PET examinations, may show similar levels of uptake, delayed imaging potentially allows differentiation of these entities by virtue of qualitative or semiquantitative differences in ${ }^{18} \mathrm{~F}$-FDG retention. Dual-phase ${ }^{18} \mathrm{~F}$-FDG PET has now been shown to improve on the accuracy of evaluation of solitary pulmonary nodules, particularly its specificity, if the combination of an early maximum SUV of greater than or equal to 2.5 and lack of washout on delayed imaging are used as the criteria for diagnosis of malignancy (10). Additional studies have shown the utility of dual-timepoint ${ }^{18}$ F-FDG PET in head and neck cancer (11), breast cancer $(12,13)$, and cervical cancer (14). There is also evidence that washout of ${ }^{18} \mathrm{~F}$-FDG over time may help to discriminate postradiation changes from residual malignancy in the setting of residual masses after radiotherapy (15). However, this discrimination can also be performed reasonably reliably using pattern recognition (16).

In the majority of these studies, a high retention index (RI) has been suggested to be a feature of cancers whereas a low $\mathrm{RI}$ is more typical of inflammatory lesions. If only things were always so simple. While working with colleagues in Singapore, where tuberculosis is significantly more prevalent than in Australia, we often came across metabolically active apical lung lesions. Although I would have confidently diagnosed lung cancer as the most likely differential diagnosis for such lesions in Australia, I was much more tempted to equivocation in Singapore. Aware of the 
data on dual-phase ${ }^{18}$ F-FDG PET, we attempted this only to find that many lesions subsequently found to be granulomatous demonstrated positive RIs. This experience was supported by a subsequent publication from Taiwan (17). Thus, it would seem that some inflammatory or granulomatous diseases can continue to trap ${ }^{18} \mathrm{~F}-\mathrm{FDG}$ rather than washing out at between 1 and $3 \mathrm{~h}$. This phenomenon has also been shown in a study attempting to differentiate carcinoma of the gallbladder from cholecystitis (18).

The current study also demonstrated some benign lesions with an RI more than double those in the primary. However, the majority of benign lesions had, as expected, an RI of less than half that in the primary. Importantly, there was a strong correlation between the RI of the primary site and the RIs of sites subsequently proven to represent metastases. Interestingly, the slope of the RI regression was greater than unity. This means that the RIs of metastatic lesions tended to be higher than those of primary lesions. Perhaps this reflects the fact that the signal from the primary tumor has a component of active inflammation, since this is not an uncommon finding in association with non-small cell lung cancer due to obstructive atelectasis. The uptake of ${ }^{18} \mathrm{~F}-\mathrm{FDG}$ at distant metastatic sites is, perhaps, more likely to be primarily due to incorporation in malignant cells. The correlation between the RIs at the primary and metastatic sites was stronger than that between early or late maximum SUV for those same lesions. This is somewhat surprising since experience has taught us that lesions with significantly different ${ }^{18}$ F-FDG avidity, unless explained by partial-volume effects, are likely to reflect a different pathology. For example, low uptake in enlarged mediastinal nodes without radiologic features of necrosis is likely to reflect reactive lymphadenopathy in the presence of intense uptake in the primary.

The data presented in the current paper provide a logistic dilemma for the busy clinical PET facility. Do all patients with possible remote sites of disease require delayed imaging? If this were to be adopted routinely, there would be major implications with respect to patient throughput and convenience. In establishing the nature of focal ${ }^{18} \mathrm{~F}-\mathrm{FDG}$ uptake abnormalities in association with a known primary malignancy, a pragmatic approach is to apply some simple tests to determine the likelihood of a metastatic basis. What is the a priori likelihood of metastatic disease in this particular patient? Is the intensity of uptake consistent with the metabolic phenotype of the known primary? Can the difference in uptake be explained by technical factors? Is the pattern and distribution of uptake abnormality that which would be expected for a metastasis from the primary? And, in the situation where most PET studies are now performed on PET/CT, does the anatomic correlation indicate a possible causality? When experienced PET readers apply such filters to their interpretation of PET, there is evidence that nonmalignant focal ${ }^{18} \mathrm{~F}$-FDG accumulations can generally be recognized as such (19). In cases where the answers to the above questions still leave the reporting physician in doubt, delayed imaging encompassing both the primary and the remote sites and calculation of their relative RIs may provide a further clue to the likelihood of malignancy. However, when the nature of the offspring is critical to future joy or pain, determination of cellular lineage is still the gold standard.

\section{Rodney J. Hicks \\ Peter MacCallum Cancer Centre and University of Melbourne Melbourne, Australia}

\section{REFERENCES}

1. Uesaka D, Demura Y, Ishizaki T, et al. Evaluation of dual-time-point ${ }^{18} \mathrm{~F}$-FDG PET for staging in patients with lung cancer. $J$ Nucl Med. 2008;49: 1606-1612.

2. Elgazzar AH, Fernandez-Ulloa M, Silberstein EB. ${ }^{201} \mathrm{Tl}$ as a tumour-localizing agent: current status and future considerations. Nucl Med Commun. 1993;14:96-103.

3. Abdel-Dayem H. ${ }^{201}$ T1-chloride uptake ratios in differentiating benign from malignant lesions: recommendations for ratio calculations and interpretation. Nucl Med Commun. 1995;16:145-149.
4. Matthies A, Hickeson M, Cuchiara A, Alavi A. Dual time point ${ }^{18}$ F-FDG PET for the evaluation of pulmonary nodules. J Nucl Med. 2002;43:871875

5. Strauss LG. Fluorine-18 deoxyglucose and falsepositive results: a major problem in the diagnostics of oncological patients. Eur J Nucl Med. 1996;23: 1409-1415.

6. Kubota R, Yamada S, Kubota K, et al. Intratumoral distribution of fluorine-18-fluorodeoxyglucose in vivo: high accumulation in macrophages and granulation tissues studied by microautoradiography. J Nucl Med. 1992;33:1972-1980.

7. Brown RS, Leung JY, Fisher SJ, et al. Intratumoral distribution of tritiated fluorodeoxyglucose in breast carcinoma: I. Are inflammatory cells important? J Nucl Med. 1995;36:1854-1861.

8. Yamada S, Kubota K, Kubota R, Ido T, Tamahashi N. High accumulation of fluorine-18-fluorodeoxyglucose in turpentine-induced inflammatory tissue. J Nucl Med. 1995;36:1301-1306.

9. Zhuang H, Pourdehnad M, Lambright ES, et al. Dual time point ${ }^{18} \mathrm{~F}$-FDG PET imaging for differentiating malignant from inflammatory processes. J Nucl Med. 2001;42:1412-1417.

10. Alkhawaldeh K, Bural G, Kumar R, Alavi A. Impact of dual-time-point ${ }^{18} \mathrm{~F}$-FDG PET imaging and partial volume correction in the assessment of solitary pulmonary nodules. Eur J Nucl Med Mol Imaging. 2008;35:246-252.

11. Hustinx R, Smith RJ, Benard F, et al. Dual time point fluorine-18 fluorodeoxyglucose positron emission tomography: a potential method to differentiate malignancy from inflammation and normal tissue in the head and neck. Eur $J$ Nucl Med. 1999;26:1345-1348.

12. Kumar R, Loving VA, Chauhan A, et al. Potential of dual-time-point imaging to improve breast cancer diagnosis with ${ }^{18}$ F-FDG PET. J Nucl Med. 2005;46:1819-1824.

13. Mavi A, Urhan M, Yu JQ, et al. Dual time point ${ }^{18}$ F-FDG PET imaging detects breast cancer with high sensitivity and correlates well with histologic subtypes. J Nucl Med. 2006;47:1440-1446.

14. Yen TC, Ng KK, Ma SY, et al. Value of dual-phase 2-fluoro-2-deoxy-d-glucose positron emission tomography in cervical cancer. J Clin Oncol. 2003; 21:3651-3658.

15. Koike I, Ohmura M, Hata M, et al. FDG-PET scanning after radiation can predict tumor regrowth three months later. Int J Radiat Oncol Biol Phys. 2003;57:1231-1238.

16. Hicks RJ, Mac Manus MP, Matthews JP, et al. Early FDG-PET imaging after radical radiotherapy for non-small-cell lung cancer: inflammatory changes in normal tissues correlate with tumor response and do not confound therapeutic response evaluation. Int J Radiat Oncol Biol Phys. 2004; 60:412-418.

17. Yen RF, Chen KC, Lee JM, et al. ${ }^{18}$ F-FDG PET for the lymph node staging of non-small cell lung cancer in a tuberculosis-endemic country: is dual time point imaging worth the effort? Eur J Nucl Med Mol Imaging. 2008;35:13051315 .

18. Nishiyama Y, Yamamoto $\mathrm{Y}$, Fukunaga K, et al. Dual-time-point ${ }^{18}$ F-FDG PET for the evaluation of gallbladder carcinoma. J Nucl Med. 2006;47: 633-638.

19. Wang G, Lau EW, Shakher R, et al. How do oncologists deal with incidental abnormalities on whole-body fluorine-18 fluorodeoxyglucose PET/ CT? Cancer. 2007;109:117-124. 